\title{
Primary Care-Based Investigation of the Effect of Sitagliptin on Blood Pressure in Hypertensive Patients With Type 2 Diabetes
}

\author{
Shouhei Yuasa ${ }^{\mathrm{a}}$, Kazuyoshi Sato ${ }^{\mathrm{a}}$, Takamoto Furuki ${ }^{\mathrm{a}}$, Kosuke Minamizawa ${ }^{\mathrm{a}}$, Hiroyuki Sakai ${ }^{\mathrm{a}}$ \\ Yuichi Numata $^{\text {a }}$, Keiichi Chin ${ }^{\text {a }}$, Jisho Kojima ${ }^{a}$, Masaaki Miyakawa ${ }^{a}$, Ikuro Matsubab, c
}

\begin{abstract}
Background: The influence of long-term sitagliptin therapy on office blood pressure (BP) and home BP has been unclear.

Methods: In a retrospective cohort study of 454 patients with type 2 diabetes, the following variables were analyzed before and at 3,6, 9, and 12 months after initiation of sitagliptin therapy: office systolic blood pressure (SBP), office diastolic blood pressure (DBP), office pulse rate, morning home SBP, morning home DBP, morning home pulse rate, evening home SBP, evening home DBP, evening home pulse rate, hemoglobin A1c (HbA1c), plasma glucose, lipid profile, and renal function parameters.
\end{abstract}

Results: The office SBP showed a significant decrease after 6 and 12 months of sitagliptin therapy ( $\mathrm{P}<0.01$ and $\mathrm{P}<0.01$, respectively), while office DBP was decreased significantly at all time points of evaluation (3, 6, 9, and 12 months: $\mathrm{P}<0.05, \mathrm{P}<0.001, \mathrm{P}<0.001$, and $\mathrm{P}<0.05$, respectively). Analysis of covariance revealed a significant decrease in office SBP after 6 and 12 months, as well as significant reduction of office DBP after 6 and 9 months. Morning home SBP and DBP were significantly reduced after 6 months, as was evening home DBP after 6 and 12 months, but there was no significant decrease in evening home SBP. HbAlc and plasma glucose levels were significantly reduced at all time points of evaluation. Examination of the lipid profile revealed that total cholesterol, low-density lipoprotein cholesterol, and triglycerides were also decreased at all time points of evaluation, while high-density lipoprotein cholesterol was significantly reduced after 3, 9, and 12 months. Significant reduction of the estimated glomerular filtration rate was observed after 6,9 , and 12 months, and the urinary albumin/creatinine ratio was significantly lower at 9 and 12 months. Serum creatinine was increased significantly at all time points of evaluation.

Manuscript accepted for publication December 02, 2016

${ }^{a}$ Hypertension and Renal Disease Committee Study Group, Kanagawa Physicians Association, Kanagawa, Japan

${ }^{b}$ Diabetes Committee Study Group, Kanagawa Physicians Association, Kanagawa, Japan

${ }^{c}$ Corresponding Author: Ikuro Matsuba, Matsuba Medical Clinic, 2-159 Tsukagoshi, Saiwai-ku, Kawasaki-shi, Kanagawa 212-0024, Japan.

Email: ikuro@matsuba-web.com

doi: https://doi.org/10.14740/jocmr2820w
Conclusions: BP was slightly but significantly reduced from 6 months after initiation of sitagliptin therapy, indicating that this antidiabetic drug has pleiotropic effects, including an antihypertensive effect.

Keywords: Hypertension; Pleiotropic effect; Dipeptidyl peptidase-4 inhibitor; Home blood pressure; Diabetes mellitus

\section{Introduction}

In the Action to Control Cardiovascular Risk in Diabetes (ACCORD) trial, patients with type 2 diabetes were assigned to an intensive therapy group $(<120 \mathrm{~mm} \mathrm{Hg})$ or a standard therapy group $(<140 \mathrm{~mm} \mathrm{Hg})$ according to the target blood pressure (BP), and no difference in fatal or non-fatal cardiovascular events was found between the two groups [1]. After publication of these findings, the target BP for patients with diabetes was relaxed in Europe and the USA. However, diabetes is believed to be a risk factor for stroke independent of gender in Japan [2], and more stringent BP control is recommended [3, 4].

Sitagliptin is a dipeptidyl peptidase-4 inhibitor that has been reported to show pleiotropic actions, including a lipidlowering effect [5], urinary albumin-lowering effect [6-8], and BP-lowering effect $[5,9,10]$. Ogawa et al [9] reported that alternate-day administration of sitagliptin reduced the systolic blood pressure (SBP) independently of its hypoglycemic effect and the decrease in body mass index (BMI). Mistry et al [10] administered sitagliptin to non-diabetic patients with mild to moderate hypertension and performed 24-h ambulatory BP monitoring, which revealed BP reduction although by only 2 $-3 \mathrm{~mm} \mathrm{Hg}$.

In the present study, we retrospectively investigated the changes of BP over a 12-month period after initiation of sitagliptin therapy using both office BP and home BP data, which are thought to possess good reproducibility.

\section{Patients and Methods}

\section{Subjects}

Patients aged 20 years or older who had been treated with sit- 
Table 1. Clinical Profile of the Subjects

\begin{tabular}{|c|c|}
\hline & Baseline \\
\hline Male/female & $251: 203$ \\
\hline Age (years) & $67.8 \pm 11.3$ \\
\hline Weight (kg) & $64.9 \pm 13.5$ \\
\hline \multicolumn{2}{|l|}{ Office BP (mm Hg) } \\
\hline SBP & $132.98 \pm 0.74$ \\
\hline DBP & $75.38 \pm 0.49$ \\
\hline \multicolumn{2}{|l|}{ Morning home BP (mm Hg) } \\
\hline SBP & $129.27 \pm 1.12$ \\
\hline DBP & $74.49 \pm 0.99$ \\
\hline \multicolumn{2}{|l|}{ Evening home BP (mm Hg) } \\
\hline SBP & $127.15 \pm 1.39$ \\
\hline DBP & $71.88 \pm 1.06$ \\
\hline Duration of diabetes (years) & $8.9 \pm 7.2$ \\
\hline Smoking history, n (\%) & $97(21.4)$ \\
\hline Drinking history, n (\%) & $148(32.6)$ \\
\hline Hypertension, n (\%) & $330(72.7)$ \\
\hline Dyslipidemia, n (\%) & $320(70.5)$ \\
\hline Cerebrovascular accident, n (\%) & $44(9.7)$ \\
\hline Myocardial infarction, n (\%) & $18(4.0)$ \\
\hline Angina, n (\%) & $41(9.0)$ \\
\hline Peripheral arterial disease, $\mathrm{n}(\%)$ & $13(2.9)$ \\
\hline Retinopathy, n (\%) & $31(6.8)$ \\
\hline Nephropathy, n (\%) & $72(15.9)$ \\
\hline Neuropathy, n (\%) & $35(7.7)$ \\
\hline
\end{tabular}

Age, weight, and duration of diabetes: mean \pm SD; office BP and home $B P$ : least squares mean \pm SE. Office BP: office blood pressure; morning home BP: morning home blood pressure; evening home BP: evening home blood pressure.

agliptin for at least 12 months were enrolled in this study after oral informed consent was obtained. The study was conducted in accordance with the Declaration of Helsinki, with the protocol being approved by the Ethics Committee of Kanagawa Physicians Association. The study period was from September 30, 2012 to October 31, 2013 and participating doctors were not diabetes specialists.

\section{Study design}

This was a retrospective study performed on subjects who received uniform management. In all subjects, the office SBP, office diastolic blood pressure (DBP), and office pulse rate (PR) were measured before the initiation of sitagliptin therapy and at 3, 6, 9, and 12 months after the start of the therapy. Morning home SBP, morning home DBP, morning home PR, evening home SBP, evening home DBP, and evening home PR were also measured. Body weight (BW), BMI, blood glucose
(BG), hemoglobin A1c (HbA1c), total cholesterol (TC), lowdensity lipoprotein cholesterol (LDL-C), high-density lipoprotein cholesterol (HDL-C), triglycerides (TG), estimated glomerular filtration rate (eGFR), and urinary albumin/creatinine ratio (UACR) were determined at the same times.

\section{Statistical analysis}

A comparison of office measurements (SBP, DBP, and PR) and home measurements (morning and evening SBP, DBP, and PR) was made between baseline and after 3, 6, 9, and 12 months of sitagliptin therapy by using a linear mixed model. If a significant difference was noted between the values, the DunnettHsu test was performed to adjust for multiple comparisons. For office SBP, office DBP, and office PR, a step-down procedure (Wald test with the level of significance set at 15\%) was performed for the following 10 baseline patient characteristics (using the time and site of measurement as fixed effects): gender, age, BMI, concomitant antihypertensive drugs, smoking, alcohol intake, dyslipidemia, fatty liver, $\mathrm{HbA1c}$, and BP before sitagliptin treatment. Each estimate was adjusted for the selected variables. Values of BW, BMI, HbAlc, BG, lipid profile, and kidney function parameters were also compared by using a linear mixed model. If a significant difference was found, the Dunnett-Hsu test was employed for adjustment. While raw data on TG and UACR did not show a normal distribution, normality was obtained after logarithmic transformation.

All analyses were performed by using SAS version 9.2. Results were expressed as the least squares mean \pm standard error (SE) or as the mean \pm standard deviation (SD) (age, BW, $\mathrm{BMI}$, and duration of diabetes), and significance was accepted at $\mathrm{P}<0.05$ (two-sided).

\section{Results}

Baseline characteristics of the subjects are shown in Table 1. A total of 530 patients were enrolled and 76 patients were excluded (38 patients whose antihypertensive therapy was changed during the study period, 37 patients whose office BP was not measured, and one patient who did not take sitagliptin). The remaining 454 patients formed the analysis population. However, data were missing for two of the 10 baseline characteristics (age and smoking) in 70 patients, and they were excluded from evaluation of covariates. Office SBP and DBP were evaluated in 444 patients and 384 patients, respectively.

BP

Office SBP showed a significant decrease at 6 and 12 months after initiation of sitagliptin therapy compared with baseline, while there was a significant decrease in office DBP at all time points of assessment (3, 6, 9, and 12 months). In contrast, there was no significant change of office PR. Analysis of covariance demonstrated that gender, concomitant antihypertensive drugs, and baseline BP were factors with a possible influence on of- 


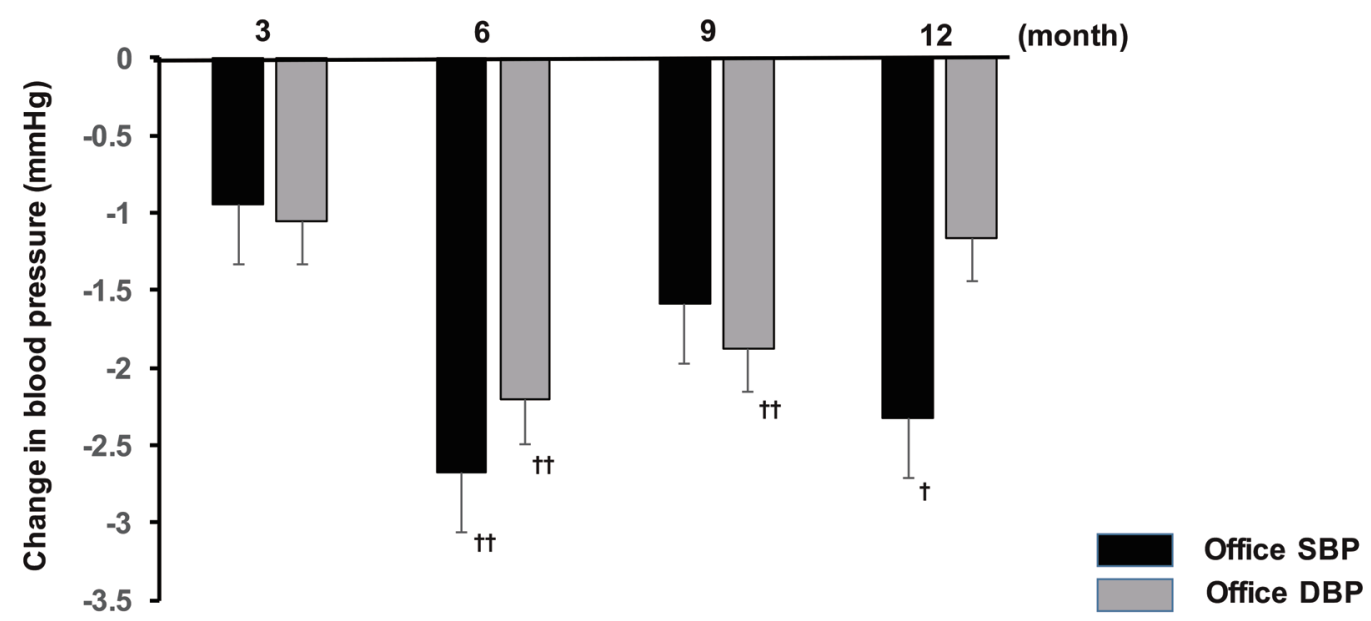

Figure 1. Changes in office BP from baseline to 12 months. ${ }^{\dagger} \mathrm{P}<0.05,{ }^{\dagger} \mathrm{P}<0.01$. SBP: systolic blood pressure; DBP: diastolic blood pressure.

fice SBP, whereas age, concomitant antihypertensive drugs, smoking, and baseline BP were factors that potentially influenced office DBP. In addition, the factors with a potential influence on office PR were fatty liver, BMI, and baseline BP. After adjustment for the above-mentioned factors, office SBP showed a significant decrease at 6 and 12 months, while office DBP was significantly lower at 6 and 9 months (Fig. 1). In addition, there was a significant decrease in morning home DBP after 6 months of sitagliptin treatment, while evening home DBP displayed a significant decrease at 6 and 12 months and morning home SBP was also significantly reduced at 6 months. However, there was no significant change of evening home SBP throughout the study period (Fig. 2), as well as no significant change of morning or evening home PR.

\section{Changes of other variables}

Levels of $\mathrm{HbAlc}$, fasting plasma glucose (FPG), and postprandial plasma glucose ( $\mathrm{PPG}$ ) were all reduced significantly at 3, 6, 9, and 12 months after the start of sitagliptin therapy, showing a maximum decrease of $-0.83 \%,-24.87 \mathrm{mg} / \mathrm{dL}$, and $-38.13 \mathrm{mg} / \mathrm{dL}$, respectively. There was no significant change of BMI at any time. Significant reduction of TC, LDL-C, and TG was noted at all time points after the start of sitagliptin treatment, while HDL-C displayed a significant decrease at 3, 9 , and 12 months. Serum creatinine was increased significantly at all time points after starting sitagliptin treatment, eGFR was decreased significantly at 6,9 , and 12 months, and UACR was significantly lower at 9 and 12 months (Table 2).

\section{Discussion}

In the present study, we found that sitagliptin reduced office SBP and DBP from 6 months after the initiation of treatment. With regard to home BP, there was significant reduction of morning SBP and DBP. Evening DBP also showed a significant decrease with sitagliptin therapy, but there was no significant change of evening SBP. The baseline BMI of the study population was $>25 \mathrm{~kg} / \mathrm{m}^{2}$ and there were many obese patients, but BW and BMI did not change after 12 months of sitagliptin
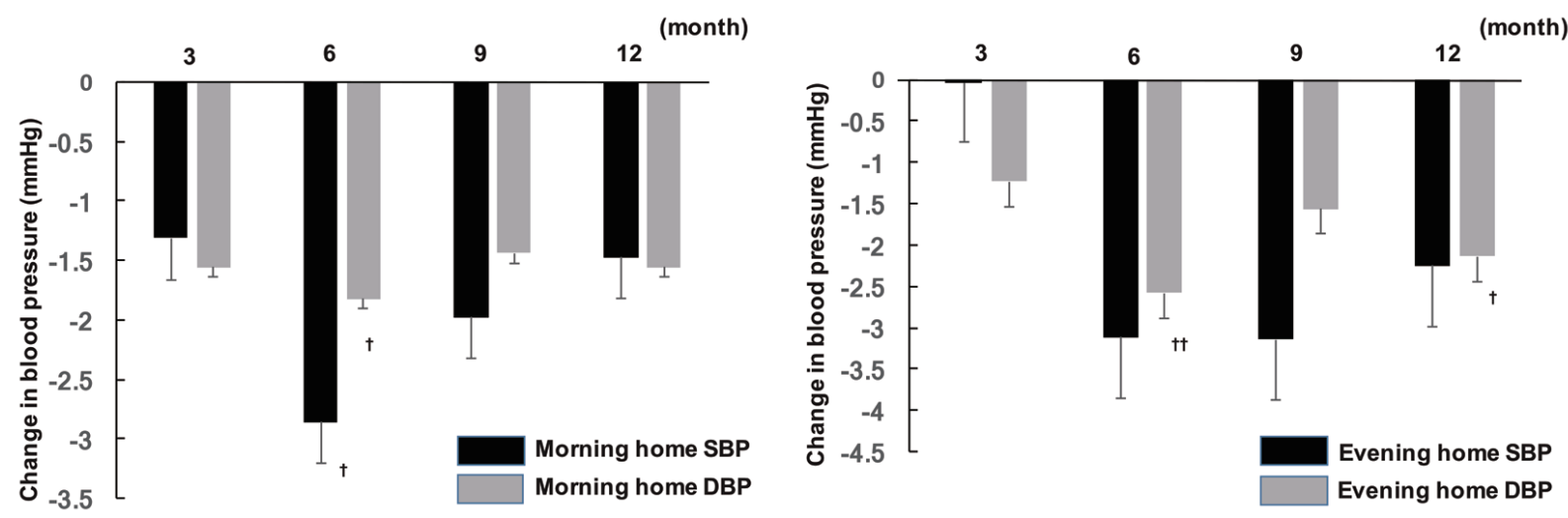

Figure 2. Changes in home BP from baseline to 12 months. ${ }^{\mathrm{t} P}<0.05$, ${ }^{\dagger} \mathrm{P}<0.01$. SBP: systolic blood pressure; DBP: diastolic blood pressure. 
Table 2. Changes of Variables From Baseline 12 Months

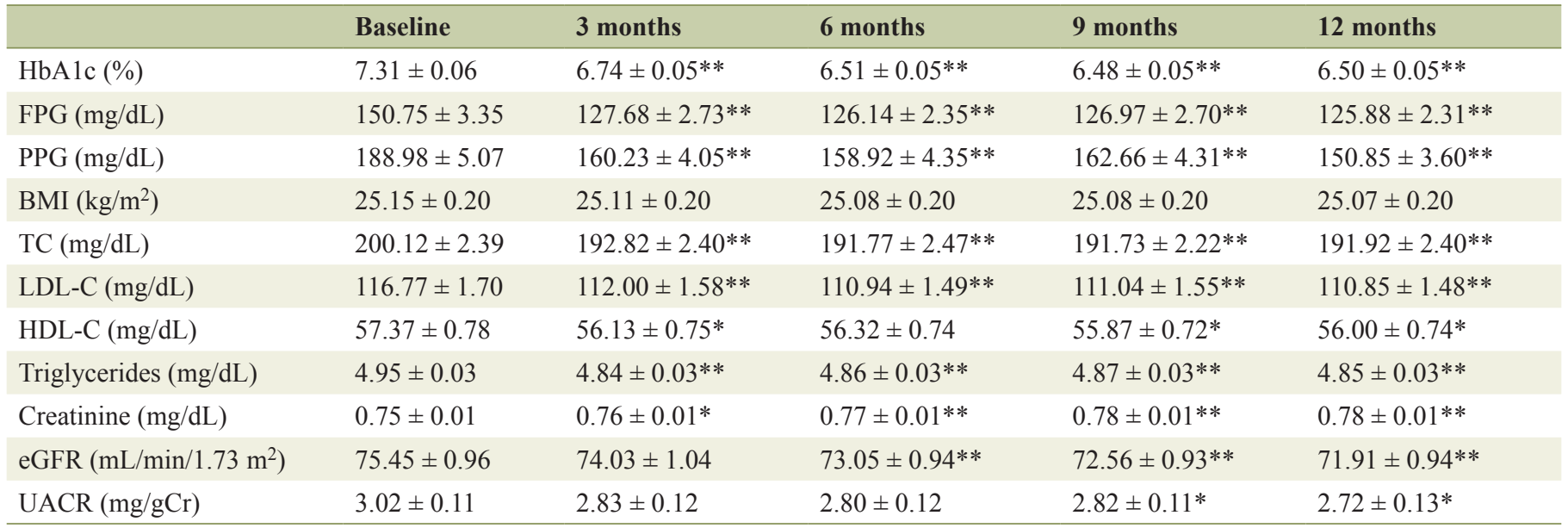

ANOVA vs. baseline, ${ }^{* *} P<0.01 ;{ }^{*} P<0.05$. Least squares mean $\pm S E$. Data on triglycerides and UACR were subjected to logarithmic transformation. HbA1c: hemoglobin A1c; FPG: fasting plasma glucose; PPG: postprandial plasma glucose; BMI: body mass index; TC: total cholesterol; LDL-C: low-density lipoprotein cholesterol; HDL-C: high-density lipoprotein cholesterol; eGFR: estimated glomerular filtration rate; UACR: urinary albumin/ creatinine ratio.

therapy, suggesting that its BP-lowering effect was not due to weight loss. In patients with type 2 diabetes, $\mathrm{Na}^{+}$reabsorption by the proximal renal tubules is increased, which causes fluid retention and thereby hypertension. It was reported that $\mathrm{Na}^{+}$ excretion by the proximal tubule was increased and GFR declined when insulin-resistant obese male patients were treated with glucagon-like polypeptide-1 [11]. The activity of glucagon-like polypeptide-1 is enhanced by sitagliptin, which suppresses $\mathrm{Na}^{+}$reabsorption from the proximal tubules via $\mathrm{Na}^{+} /$ $\mathrm{H}^{+}$exchanger isoform 3 and contributes to BP reduction [12]. In the present study, SBP and DBP were reduced and eGFR was significantly decreased from 6 months after initiation of sitagliptin therapy. If the BP-lowering effect of sitagliptin was associated with increased urinary $\mathrm{Na}^{+}$excretion ( $\mathrm{Na}$ diuresis), it should have been seen early in the treatment period, but SBP and DBP both decreased from 6 months onward. This finding suggests that the antihypertensive effect of sitagliptin may be exerted by a different mechanism rather than Na diuresis; however, urinary $\mathrm{Na}^{+}$was not measured in our study, and we could not provide evidence for this hypothesis. Recently, Kim et al reported that glucagon-like polypeptide-1 was involved in $\mathrm{Na}$ diuresis through elevation of atrial natriuretic peptide levels in animals [13].

To improve outcomes for patients with diabetes, comprehensive control of BG, BP, and lipids is important, and suppressing urinary albumin excretion is also a key point. Several studies have demonstrated that sitagliptin decreases urinary albumin excretion [6-8], but the mechanism involved has not been clarified. Groop et al [14] reported that the urinary albumin-lowering effect of a similar drug (linagliptin) was not related to changes of BG or BP. On the other hand, Kawasaki et al [8] reported the changes of SBP and eGFR were prognostic indicators of the decrease in urinary albumin. In the present study, eGFR decreased slightly over time and urinary albumin excretion was reduced at 9 months after initiation of sitagliptin therapy. A decrease in eGFR due to sitagliptin therapy reflects a reduced functional intraglomerular pressure, and can be used as a clinical indicator that the treatment is likely to also reduce $\mathrm{BP}$ and urinary albumin.

In the present study, the maximum reduction of both office SBP (2.7 $\mathrm{mm} \mathrm{Hg}$ ) and office DBP (2.2 $\mathrm{mm} \mathrm{Hg}$ ) was noted after 6 months of sitagliptin therapy. The mean reduction of BP was small, in agreement with other reports on the BP-lowering effect of sitagliptin [5,9]. According to a recent systematic review and meta-analysis [15], reduction of SBP by $10 \mathrm{~mm} \mathrm{Hg}$ is associated with a $20 \%$ decrease in cardiovascular events and $13 \%$ decline in the all-cause death rate. Although the clinical implications of a slight reduction of BP by $2-3 \mathrm{~mm} \mathrm{Hg}$ with sitagliptin therapy are not clear, an oral glucose-lowering agent that also has an antihypertensive effect should be desirable for suppressing cardiovascular events in diabetic patients with hypertension.

To the best of our knowledge, there have been no previous investigations into the effect of sitagliptin on home BP. We assessed home BP in the present study and found significant decreases in morning SBP, morning DBP, and evening DBP with sitagliptin treatment, but evening SBP did not change significantly throughout the 12-month study period. A possible explanation for this finding is that the baseline home BP levels were slightly lower than the office BP levels, since analysis of covariance showed that the baseline office SBP and DBP had an influence on the antihypertensive effect of sitagliptin therapy. That is, the antihypertensive effect of sitagliptin was stronger when the baseline BP was higher. Another possible factor is that home BP was not measured in a large enough number of patients.

\section{Limitations}

This study had several limitations. First, it was a retrospective cohort investigation and the results were inevitably influ- 
enced by various confounders. A large-scale intervention study would be needed to confirm the relationship between sitagliptin and BP. Second, we did not measure urinary $\mathrm{Na}^{+}$excretion, which means that we could not clearly explain the mechanism by which sitagliptin exhibits an antihypertensive effect. However, urinary $\mathrm{Na}^{+}$excretion is influenced by dietary salt intake and other factors that confound the interpretation of changes in excretion caused by sitagliptin, if any.

\section{Conclusion}

In the present study, we showed that standard sitagliptin therapy was associated with a significant decrease in office BP (both SBP and DBP) and most home BP parameters (morning SBP, morning DBP, and evening DBP). Sitagliptin also reduced BG, HbA1c, and lipid parameters (TC, LDL-C, and TG), confirming the pleiotropic effects of this medication. Further studies will be needed to determine how improvement in these surrogate markers is related to reduction of cardiovascular events.

\section{Acknowledgments}

We would also like to thank all the ATTEST-K study group investigators who contributed to this research, as well as the Hypertension and Renal Disease Committee Study Group of the Kanagawa Physicians Association.

\section{Grant Support}

This research was supported by funds from the Japan Kidney Foundation and a grant from the Japan Renal Foundation.

\section{Conflicts of Interest}

The authors declare no conflicts of interest.

\section{References}

1. Cushman WC, Evans GW, Byington RP, Goff DC, Jr., Grimm RH, Jr., Cutler JA, Simons-Morton DG, et al. Effects of intensive blood-pressure control in type 2 diabetes mellitus. N Engl J Med. 2010;362(17):1575-1585.

2. Doi Y, Ninomiya T, Hata J, Fukuhara M, Yonemoto K, Iwase M, Iida M, et al. Impact of glucose tolerance status on development of ischemic stroke and coronary heart disease in a general Japanese population: the Hisayama study. Stroke. 2010;41(2):203-209.

3. Bangalore S, Kumar S, Lobach I, Messerli FH. Blood pressure targets in subjects with type 2 diabetes mellitus/impaired fasting glucose: observations from tra- ditional and bayesian random-effects meta-analyses of randomized trials. Circulation. 2011;123(24):2799-2810, 2799 p following 2810 .

4. Reboldi G, Gentile G, Angeli F, Ambrosio G, Mancia G, Verdecchia P. Effects of intensive blood pressure reduction on myocardial infarction and stroke in diabetes: a meta-analysis in 73,913 patients. J Hypertens. 2011;29(7):1253-1269.

5. Kubota A, Maeda H, Kanamori A, Matoba K, Jin Y, Minagawa F, Obana M, et al. Pleiotropic effects of sitagliptin in the treatment of type 2 diabetes mellitus patients. J Clin Med Res. 2012;4(5):309-313.

6. Hattori S. Sitagliptin reduces albuminuria in patients with type 2 diabetes. Endocr J. 2011;58(1):69-73.

7. Mori H, Okada Y, Arao T, Tanaka Y. Sitagliptin improves albuminuria in patients with type 2 diabetes mellitus. J Diabetes Investig. 2014;5(3):313-319.

8. Kawasaki I, Hiura Y, Tamai A, Yoshida Y, Yakusiji Y, Ikuno Y, Okada M, et al. Sitagliptin reduces the urine albumin-to-creatinine ratio in type 2 diabetes through decreasing both blood pressure and estimated glomerular filtration rate. J Diabetes. 2015;7(1):41-46.

9. Ogawa S, Ishiki M, Nako K, Okamura M, Senda M, Mori T, Ito S. Sitagliptin, a dipeptidyl peptidase-4 inhibitor, decreases systolic blood pressure in Japanese hypertensive patients with type 2 diabetes. Tohoku J Exp Med. 2011;223(2):133-135.

10. Mistry GC, Maes AL, Lasseter KC, Davies MJ, Gottesdiener KM, Wagner JA, Herman GA. Effect of sitagliptin, a dipeptidyl peptidase-4 inhibitor, on blood pressure in nondiabetic patients with mild to moderate hypertension. J Clin Pharmacol. 2008;48(5):592-598.

11. Gutzwiller JP, Tschopp S, Bock A, Zehnder CE, Huber AR, Kreyenbuehl M, Gutmann H, et al. Glucagon-like peptide 1 induces natriuresis in healthy subjects and in insulin-resistant obese men. J Clin Endocrinol Metab. 2004;89(6):3055-3061.

12. Pacheco BP, Crajoinas RO, Couto GK, Davel AP, Lessa LM, Rossoni LV, Girardi AC. Dipeptidyl peptidase IV inhibition attenuates blood pressure rising in young spontaneously hypertensive rats. J Hypertens. 2011;29(3):520528.

13. Kim M, Platt MJ, Shibasaki T, Quaggin SE, Backx PH, Seino S, Simpson JA, et al. GLP-1 receptor activation and Epac2 link atrial natriuretic peptide secretion to control of blood pressure. Nat Med. 2013;19(5):567-575.

14. Groop PH, Cooper ME, Perkovic V, Emser A, Woerle HJ, von Eynatten M. Linagliptin lowers albuminuria on top of recommended standard treatment in patients with type 2 diabetes and renal dysfunction. Diabetes Care. 2013;36(11):3460-3468.

15. Ettehad D, Emdin CA, Kiran A, Anderson SG, Callender T, Emberson J, Chalmers J, et al. Blood pressure lowering for prevention of cardiovascular disease and death: a systematic review and meta-analysis. Lancet. 2016;387(10022):957-967. 